\title{
Methionine Requirement of Sporulation in a Streptomyces fradiae Mutant
}

\author{
By GYÖRGY VARGHA, ${ }^{1}$ VALÉRIA Zs.-NAGY, ${ }^{2}$ GYÖRGY LUSTYIK ${ }^{2}$ AND \\ GÁBOR SZABÓ ${ }^{1 *}$ \\ Department of Biology and Fritz Verzár International Laboratory for Experimental \\ Gerontology ${ }^{2}$, University Medical School, H-4012 Debrecen, Hungary
}

(Received 31 December 1985; revised 16 June 1986)

\begin{abstract}
When an Arg- mutant of Streptomyces fradiae was grown on sucrose-nitrate agar supplemented with L-citrulline, it produced only non-sporulating aerial mycelia. Sporulation could be induced by adding 0.1 to $10.0 \mathrm{mM}$-L-methionine or $0.01 \mathrm{mM}$-cyanocobalamin; several other compounds including homocysteine, other amino acids, vitamins and precursors of nucleic acids were ineffective. Growth of the mutant did not differ significantly with or without methionine. The mutant was more sensitive to L-norleucine (a methionine analogue) than the parent strain. Spontaneous mutants, resistant to this analogue, were obtained. They all sporulated well without exogenously added methionine. In medium-exchange experiments, methionine was required for sporulation only after $48 \mathrm{~h}$ cultivation. The phenomenon reported here is most probably based on partially impaired methionine biosynthesis caused by an as yet uncharacterized nonauxotrophic mutation, since $\mathrm{Arg}^{+}$revertants retained their methionine requirement for sporulation, and were also sensitive to L-norleucine.
\end{abstract}

\section{INTRODUCTION}

The developmental process in Streptomyces, i.e. the formation of aerial mycelia and spores, is significantly affected by the nitrogen source in the culture medium (see reviews by Kalakoutskii \& Agre, 1976; Ensign, 1978). In Streptomyces clavuligerus (Aharonowitz \& Demain, 1979) and Streptomyces viridochromogenes (Coleman \& Ensign, 1982) it has also been shown that sporulation is regulated by the amino acid composition of the growth medium.

In Bacillus subtilis the initiation of sporulation by partial amino acid starvation involves the stringent response (Ochi et al., 1981); methionine analogues, however, induce sporulation by a different mechanism (Ochi \& Freese, 1982). In Myxococcus xanthus both methionine and its analogue DL-norleucine inhibit differentiation (Clarke \& Napier, 1983). Methionine is required for the formation of aerial mycelium in a thermophilic actinomycete referred to as Micromonospora vulgaris (Webley, 1958); it also induces arthrospore formation in the fungus Cephalosporium acremonium (Martin \& Demain, 1980).

Developmental mutants in Streptomyces were thoroughly analysed by Chater \& Merrick (1976). However, it is difficult to determine the functions of the different genes. Mutants that show specific requirements for differentiation but not for growth are interesting because they may serve as a model for investigating the metabolic basis of differentiation. We obtained a mutant of Streptomyces fradiae that apparently was not a methionine auxotroph, but which required methionine for sporulation. This paper describes the properties of this mutant.

\section{METHODS}

Organisms. Streptomyces fradiae strain St $3110\left(\mathrm{Arg}^{-} \mathrm{Str}^{\mathrm{r}}\right)$ and its parent strains M2-21 (Arg $\left.{ }^{+} \mathrm{Str}^{\mathrm{r}}\right)$ and LN 47 1Gl $\left(\mathrm{Arg}^{+} \mathrm{Str}^{\mathrm{S}}\right)$ were described by Vargha et al. (1983). Prototrophic revertants of strain St 3110 (Vargha \& Szabó, 1984) were RASP 1 and RASP 2 (spontaneous), and RAUV 1 and RAUV 2 (UV-induced). 
Growth conditions. SNA (sucrose-nitrate agar; Waksman, 1967) was used throughout in the present work. Supplements were added at $1 \mathrm{mM}$ final concentration, or as otherwise indicated. Plates were incubated at $30^{\circ} \mathrm{C}$. Usually, plates were inoculated with spores derived from surface cultures grown on SNA slants. Inocula from strain St 3110 were made as follows: $24 \mathrm{~h}$ liquid cultures grown in a medium containing $\left(\mathrm{g} \mathrm{l}^{-1}\right)$ Tryptone (Oxoid) (10), yeast extract (Oxoid) (5), $\mathrm{NaCl}(10)$ and glucose (20), $\mathrm{pH} 7 \cdot 2$, were sedimented by centrifugation; mycelia were washed with saline $(0.85 \% \mathrm{NaCl})$, sedimented again, resuspended in saline containing $25 \%(\mathrm{v} / \mathrm{v})$ glycerol and stored at $-20^{\circ} \mathrm{C}$. Later, spores of strain St 3110 grown on SNA slants supplemented with methionine were used for inoculation of the plates.

Gradient test. The method was essentially as described by Vargha et al. (1983) and Vargha \& Szabó (1984). Usually, $0.2 \mathrm{ml}$ of either a $200 \mathrm{~mm}$ solution (for most of the test compounds), or a $20 \mathrm{~mm}$ solution (for constituents of nucleic acids), or a $2 \mathrm{~mm}$ solution (for vitamins) was put into holes on agar plates. The concentrations of some solutions differed from this protocol. These were: factor $\mathrm{A}, 1 \mu \mathrm{g} \mathrm{ml}^{-1}$; seleno-DL-methionine, $0 \cdot 2 \mathrm{mg} \mathrm{ml}^{-1}$; EDTA, $50 \mathrm{~mm}$; sodium phosphate buffer, $0.2 \mathrm{M}, \mathrm{pH} 7 \cdot 2$; Tris buffer, $0.1 \mathrm{M}, \mathrm{pH} 8.5 ; \mathrm{CaCl}_{2}, 10 \mathrm{~mm} ; \mathrm{MgCl}_{2}, 10 \mathrm{~mm}$; cobalt(II) acetate, 1 or $100 \mathrm{~mm} ; \mathrm{ZnSO}_{4}, 100 \mathrm{~mm}$; and those listed by Vargha \& Szabó (1984). In some cases, simple spot-tests were made, as with DL-homoserine, DL-homocysteine, DL-ethionine, $S$-adenosyl-L-methionine (SAM), trimethoprim and methotrexate. Each solution was neutralized before use.

Growth measurements. Samples of an S. fradiae St 3110 spore suspension were filtered onto Sartorius cellulose nitrate membrane filters $(0.45 \mu \mathrm{m}$ pore size, $47 \mathrm{~mm}$ diameter), then placed on $6 \mathrm{~cm}$ diameter agar plates $(15 \mathrm{ml}$ medium). After 24,48 or $72 \mathrm{~h}$ incubation at $30^{\circ} \mathrm{C}$, the filters were removed from the plates. Mycelia were scraped off the filters, suspended in saline, and disrupted by $9 \times 20 \mathrm{~s}$ sonic treatment $(20 \mathrm{kHz}$, Braunsonic $300 \mathrm{~S}$ instrument) in an ice-bath. Proteins were precipitated with cold $5 \% \mathrm{TCA}$, dissolved in $1 \mathrm{M}-\mathrm{NaOH}$, diluted tenfold with distilled $\mathrm{H}_{2} \mathrm{O}$ and assayed by both the Lowry method and the micro-biuret method (Itzhaky \& Gill, 1964) using bovine serum albumin as standard. The filter discs with remnants of the mycelia were cut into small pieces and suspended in saline. After sonic treatment the solid phase was separated by centrifugation, washed with saline and sedimented again. The supernatants were pooled and used for protein determination (see above). The protein content of the scraped-off mycelia and that of the remnants were summed to determine growth. Non-inoculated filters were used as controls.

Medium exchange experiments. Spores of $S$. fradiae were filtered onto Sartorius cellulose membrane filters $(0.2 \mu \mathrm{m}$ pore size $)$; the filters were then placed on pairs of SNA plates. Both plates of a pair contained citrulline, but only one was supplemented with methionine. The plates were incubated at $30^{\circ} \mathrm{C}$. At intervals a pair of filters was exchanged between the two plates and further incubated; the aerial mycelia were then viewed by phasecontrast microscopy.

Microscopy. Stereomicroscopy of the colonies and phase-contrast microscopy of the mycelia were done as described by Vargha \& Szabó (1984). For scanning electron photomicrographs, fixation and dehydration were as described by Coleman \& Ensign (1982). The agar blocks were then dried with diethyl ether, coated with platinum and viewed with a JEOL model JSM 35C scanning electron microscope.

Chemicals. DL-Ethionine, seleno-DL-methionine, DL-homoserine, DL-homocysteine and $S$-adenosyl-L-methionine were from Sigma, glutathione, guanosine, glycerol and lecithin from Merck, L-norleucine from Fluka, methotrexate from Lederle Parenterals, ADP, ATP, GTP and EDTA from Reanal (Budapest) and trimethoprim from EGIS (Budapest). Factor A was a gift of U. Gräfe. The origins of the other chemicals were listed by Vargha \& Szabó (1984).

\section{RESULTS}

\section{Incomplete differentiation on citrulline-containing media}

When SNA plates containing $1 \mathrm{mM}-\mathrm{L}$-citrulline were inoculated with strain St 3110 , vegetative and aerial growth seemed to be normal (Vargha et al., 1983), but the aerial mycelia always lacked spores. This was confirmed several times by phase-contrast microscopy (Vargha \& Szabó, 1984) or scanning electron microscopy (Fig. $1 a$ ). Even when a citrulline gradient was prepared in the plate (Vargha et al., 1983), no citrulline concentration was found that supported sporulation. Cultivation for up to three weeks also failed to produce sporulation on the above media.

\section{Induction of sporulation by methionine or cyanocobalamin}

To check whether incomplete differentiation was caused by some metabolic shortage, several compounds were tested for their ability to induce sporulation in cultures grown on citrullinesupplemented synthetic medium. Among them there were simple chemicals $(\mathrm{HCl}, \mathrm{NaOH}$, bivalent cations), amino acids, intermediates of the arginine biosynthetic pathway, polyamines, 

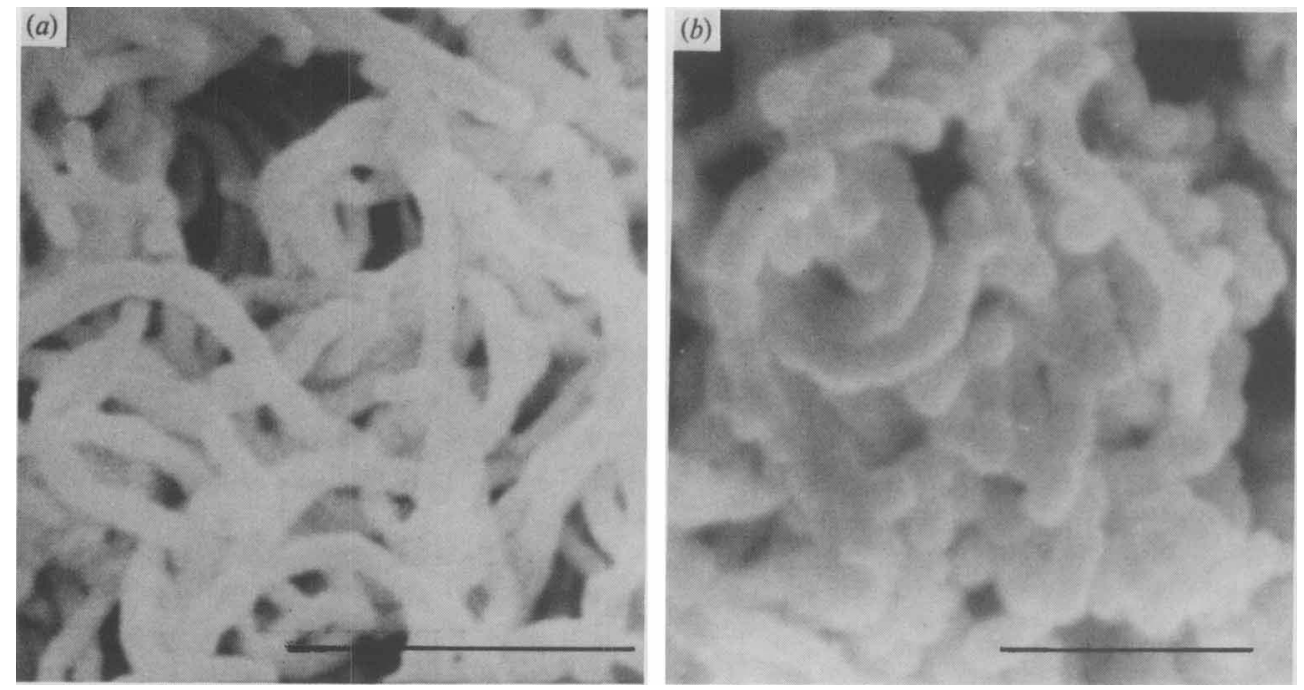

Fig. 1. Scanning electron micrographs of the aerial mycelia of $S$. fradiae St 3110. Mycelia were grown on SNA plates supplemented with $1 \mathrm{mM}$-L-citrulline $(a)$, or $1 \mathrm{mM}$-L-citrulline $+1 \mathrm{mM}$-L-methionine $(b)$. Preparations for scanning electron microscopy were made from 10-d-old cultures. Bars, $10 \mu \mathrm{m}(a)$ and $5 \mu \mathrm{m}(b)$.

components of nucleic acids, vitamins (including folate), sugars, intermediates of the Krebs cycle, lipotropic factors (choline, myo-inositol, lecithin, ethanol, Tween-40), metabolic inhibitors (ethionine, selenomethionine, trimethoprim, methotrexate) and regulatory compounds (cAMP and factor A). Of the compounds tested, only L-methionine and cyanocobalamin (vitamin $B_{12}$ ) were able to promote sporulation. When the same synthetic medium was supplemented with L-methionine, characteristic helical spore chains were formed as observed by scanning electron microscopy (Fig. 1b) or phase-contrast microscopy (not shown). This effect was specific for methionine; homoserine, homocysteine, and cysteine (intermediates of the methionine biosynthetic pathway) and SAM were ineffective. The methionine concentration needed for sporulation was at least $0.1 \mathrm{mM}$ at the time of inoculation. The same effect was observed for concentrations up to $10 \mathrm{mM}$. Cyanocobalamin $(0.002-0.01 \mathrm{mM}$ at the time of inoculation) was also able to induce sporulation on citrulline-containing medium, but this effect was completely abolished when the phosphate concentration in the medium was raised to $50 \mathrm{~mm}$. The sporulation induced by methionine was more abundant and could not be prevented by this concentration of phosphate. It is worth mentioning that methionine sulphoximine, an inducer of aerial growth in the same strain on SNA containing $1 \mathrm{mm-arginine} \mathrm{(Vargha} \mathrm{\&} \mathrm{Szabó,}$ 1984) failed to promote sporulation on medium containing $1 \mathrm{~mm}$-citrulline.

\section{Effect of methionine on growth}

Growth of mutant St 3110 was not restricted in the absence of exogenously added methionine. In order to measure biomass on solid medium the mutant was grown on membrane filters, and the protein contents of the cultures were determined. Growth in the presence of exogenous methionine did not differ significantly from when this amino acid was absent: 1 mM-methionine increased growth by only about $10 \%$ after $72 \mathrm{~h}$ cultivation. Methionine auxotrophs did not grow at all on the methionine-free medium (data not shown).

\section{Isolation and properties of analogue-resistant mutants}

Growth of mutant St 3110 was inhibited by $40 \mu \mathrm{M}$-L-norleucine, a methionine analogue. The parent strains M2-21 and LN 47 1-Gl, however, were fully resistant, even to $80 \mu \mathrm{M}$-L-norleucine. 
The inhibition could be overcome by adding methionine to the medium. When spores from a single colony of mutant St 3110 were inoculated onto SNA + citrulline + norleucine $(40 \mu \mathrm{M})$ plates, resistant colonies arose spontaneously at a frequency of $3.1 \times 10^{-5}$. Around these colonies, haloes of secondary growth appeared. The 51 resistant variants were stable on subculturing in the absence of norleucine. They retained both the $\mathrm{Arg}^{-}$and the streptomycin resistance traits of strain St 3110 . The most important feature of the norleucine resistant mutants was that - in contrast to strain St 3110 - they sporulated normally on SNA + citrulline plates lacking methionine. In this respect they behaved like strains M2-21 and LN 47 1-Gl.

\section{Determination of the time when methionine should be present in order to promote sporulation in mutant St 3110}

In order to exchange the medium in surface cultures, mycelia were grown on membrane filters placed on agar plates. The colonies were always transferred to a medium which had previously supported growth of a culture of the same age, in order to avoid the influence of fri sh nutrients. When colonies were first grown in the presence of methionine for $48 \mathrm{~h}$, then transferred to a methionine-free medium, no sporulation was observed. (Aerial mycelia usually start developing after $48 \mathrm{~h}$ cultivation.) When colonies were grown without methionine, and supplied with it later, they responded to methionine as late as the seventh day of cultivation, but the intensity of the response (i.e. sporulation) decreased after the third day. Therefore, the optimal period for the induction of spore formation by methionine was found to be between 48 and $96 \mathrm{~h}$, i.e. during the visible aerial development.

\section{Properties of the prototrophic revertants}

In order to learn whether the $\mathrm{Arg}^{-}$mutation in strain St 3110 was related to the methioninerequirement for sporulation, prototrophic revertants were examined. The isolation of $\mathrm{Arg}^{+}$ revertants was reported by Vargha \& Szabó (1984). The revertants retained the streptomycin resistance trait. When they were grown on SNA plates supplemented with $1 \mathrm{~mm}$-citrulline, only non-sporulating aerial mycelia were produced. Sporulation could be induced in all four revertants by adding methionine to the culture medium - similarly to the $\mathrm{Arg}^{-}$parent - showing that this feature was not linked to the $\mathrm{Arg}^{-}$trait. The norleucine sensitivity of the revertants was also similar to that of strain St 3110 .

\section{DISCUSSION}

Methionine or its metabolites appear to be connected with the control of differentiation in some prokaryotic organisms including an actinomycete strain (Webley, 1958; Ochi \& Freese, 1982; Clarke \& Napier, 1983), but methionine-induced fungal arthrospore formation has also been observed (Martin \& Demain, 1980). The role of methionine is not clear, and it is difficult to come to general conclusions. In some cases, methionine induced or was required for sporulation (Webley, 1958; Martin \& Demain, 1980; this report), whereas in B. subtilis sporulation was induced by methionine analogues (Ochi \& Freese, 1982). In $M$. xanthus both methionine and its analogue norleucine inhibited fruiting-body formation (Clarke \& Napier, 1983). The most informative study in this respect was by Ochi \& Freese (1982) who found that a decrease in SAM synthetase activity increased the probability of spontaneous sporulation in $B$. subtilis.

It seems that a methionine-linked regulatory mechanism of sporulation - if it exists - is present or manifests itself only in certain organisms or mutants. If so, the mutation reported here could be of use in investigating this regulation. One of the most attractive features of the mutant is that methionine is needed specifically for sporulation, and not for growth. Furthermore, methionine was ineffective when present only during the vegetative growth phase (the first $2 \mathrm{~d}$ ). It was needed for sporulation essentially during the development of aerial hyphae. Because of its specific nutritional requirement for the developmental process, the mutant can be termed a 'develotroph' or 'biototroph' - in contrast with an 'auxotroph'. The mutation leading to this 'biototrophic' ('develotrophic') phenotype was independent of the $\mathrm{Arg}^{-}$trait because the mutant phenotype reverted independently. The reversion rate of the 'develotrophic' mutation was relatively high. The cause of this has not yet been elucidated. 
The most plausible explanation of the data is that there is a partial block in the methionine biosynthetic pathway of the mutant. This would also explain its norleucine sensitivity. Methionine synthesis could be adequate for growth, but during aerial development synthesis would become insufficient, at least for sporulation. This insufficiency may be relative if cells need more methionine during sporulation than for growth, or could be a consequence of a (regulative) change in the biosynthetic pathway during differentiation.

The impaired step in the biosynthetic pathway is probably the last one, the conversion of homocysteine to methionine, since intermediates before this step were not able to mimic the effect of methionine. In addition, this is a reaction for which methylcobalamin, the active in vivo derivative of cyanocobalamin, is a cofactor, and cyanocobalamin also induced sporulation in the mutant. Alternatively, it is also possible that the mutation primarily impairs the biosynthesis of cobalamin, but this seems less probable, because the effect of cyanocobalamin on sporulation is phosphate-sensitive and weaker (fewer spores are produced: data not shown) in comparison with methionine.

It has long been known that cobalt can be favourable for the sporulation of Streptomyces strains including $S$. fradiae (Hickey \& Tresner, 1952). According to our data cobalt could act via incorporation into cobalamin, thus increasing methionine synthesis. Recently, Gräfe et al. (1985) found cobalt to have a synergistic effect with A-factor on aerial mycelium formation in $S$. griseus.

The considerable specificity of the methionine requirement for sporulation is important when considering the mechanism of action. Reactions specific to methionine are of interest. The data of Ochi \& Freese (1982) suggested the importance of the SAM pool for sporulation. Freese (1984) hypothesized that changes in DNA methylation would be an early event during initiation of sporulation in B. subtilis. In Streptomyces, Ochi et al. (1984) presented an example where exogenously supplied SAM increased antibiotic production, indicating that SAM may be involved in secondary metabolism also. The role of SAM as a possible common regulator in differentiation and secondary metabolism requires further investigation to help define the role of methionine in sporulation.

The authors thank Professor D. A. Hopwood for providing some fine chemicals. The technical assistance of Mrs Ibolya Szekeres is also acknowledged.

\section{REFERENCES}

Aharonowitz, Y. \& Demain, A. L. (1979). Nitrogen nutrition and regulation of cephalosporin production in Streptomyces clavuligerus. Canadian Journal of Microbiology 25, 61-67.

Chater, K. F. \& Merrick, M. J. (1976). Approaches to the study of differentiation in Streptomyces coelicolor A3(2). In Second International Symposium on Genetics of Industrial Microorganisms, pp. 583593. Edited by K. D. MacDonald. New York: Academic Press.

Clarke, C. H. \& NAPIER, I. (1983). Inhibitors of fruiting-body formation and challenge time experiments in Myxococcus xanthus. Journal of General Microbiology 129, 117-122.

Coleman, R. H. \& Ensign, J. C. (1982). Regulation of formation of aerial mycelia and spores of Streptomyces viridochromogenes. Journal of Bacteriology 149, 1102-1111.

ENSIGN, J. C. (1978). Formation, properties and germination of actinomycete spores. Annual Review of Microbiology 32, 185-219.

FREESE, E. (1984). Initiation of sporulation by guanine deprivation of bacilli and yeast. In Physiology of Microbial Growth and Differentiation (Proceedings of the Reinhardsbrunn Symposium, May 20-26, 1984, GDR).
GrÄFE, U., ERITT, I. \& RIESENBERG, D. (1985). Synergistic effect of cobalt on the induction by Afactor of the formation of aerial mycelium and anthracyclines by a blocked mutant of Streptomyces griseus. Journal of Basic Microbiology 25, 279-283.

HickeY, R. J. \& Tresner, H. D. (1952). A cobalt containing medium for sporulation of Streptomyces species. Journal of Bacteriology 64, 891-892.

ITZHAKI, R. F. \& GILL, D. M. (1964). A micro-biuret method for estimating proteins. Analytical Biochemistry 9, 401-410.

Kalakoutskit, L. V. \& Agre, N. S. (1976). Comparative aspects of development and differentiation in actinomycetes. Bacteriological Reviews 40, 469524.

Martin, J. F. \& Demain, A. L. (1980). Control of antibiotic biosynthesis. Microbiological Reviews 44, 230-251.

OCHI, K. \& Freese, E. (1982). A decrease in $S$ adenosylmethionine synthetase activity increases the probability of spontaneous sporulation. Journal of Bacteriology 152, 400-410.

Ochi, K., Kandala, J. C. \& Freese, E. (1981). Initiation of Bacillus subtilis sporulation by the stringent response to partial amino acid deprivation. Journal of Biological Chemistry 256, 6866-6875. 
OCHI, K., SaIto, Y., Umehara, K., Ueda, I. \& KOHSAKA, M. (1984). Restoration of aerial mycelium and antibiotic production in a Streptomyces griseoflavus arginine auxotroph. Journal of General Microbiology 130, 2007-2013.

VARGHA, G. \& Szabó, G. (1984). Nutritional requirements for differentiation in a Streptomyces fradiae mutant. Zeitschrift für allgemeine Mikrobiologie 24, 189-199.

Vargha, G., Karsai, T. \& Szabó, G. (1983). A conditional aerial mycelium-negative mutant of
Streptomyces fradiae with deficient ornithine carbamoyltransferase activity. Journal of General Microbiology 129, 539-542.

W aksman, S. A. (1967). The Actinomycetes. A Summary of Current Knowledge, p. 245. New York: Ronald Press.

WEBLEY, D. M. (1958). A defined medium for the growth of the thermophilic actinomycete Micromonospora vulgaris. Journal of General Microbiology 19, 402-406. 